KYUNGPOOK Math. J. 55(2015), 731-739

http://dx.doi.org/10.5666/KMJ.2015.55.3.731

pISSN 1225-6951 eISSN 0454-8124

(C) Kyungpook Mathematical Journal

\title{
On a Type of Semi-Symmetric Non-Metric Connection on Rie- mannian Manifolds
}

\author{
AJIT BARMAN \\ Department of Mathematics, Assistant Professor, Kabi Nazrul Mahavidyalaya, P. \\ O.-Sonamura-799181, Dist.- Sepahijala, Tripura, India \\ e-mail : ajitbarmanaw@yahoo.in
}

Abstract. The object of the present paper is to characterize a Riemannian manifold admitting a type of semi-symmetric non-metric connection.

\section{Introduction}

In 1924, Friedmann and Schouten [1] introduced the idea of semi-symmetric connection on a differentiable manifold. A linear connection $\bar{\nabla}$ on a differentiable manifold $\left(M^{n}, g\right)$ with Riemannian connection $\nabla$ is said to be a semi-symmetric connection if the torsion tensor $T$ of the connection $\bar{\nabla}$ satisfies

$$
T(X, Y)=\eta(Y) X-\eta(X) Y,
$$

where $\eta$ is a 1 -form and $\xi$ is a vector field defined by $\eta(X)=g(X, \xi)$, for all vector fields $X \in \chi\left(M^{n}\right), \chi\left(M^{n}\right)$ is the set of all differentiable vector fields on $M^{n}$.

In 1932, Hayden [4] introduced the idea of semi-symmetric connections on a Riemannian manifold $\left(M^{n}, g\right)$. A semi-symmetric connection $\bar{\nabla}$ is said to be a semi-symmetric metric connection if

$$
\bar{\nabla} g=0 .
$$

The study of semi-symmetric metric connection was further developed by Yano [6], Amur and Pujar [7], Chaki and Konar [12], De [17] and many others.

Received June 26, 2013; accepted December 13, 2013.

2010 Mathematics Subject Classification: 53C25.

Key words and phrases: Semi-symmetric metric connection, almost pseudo symmetric manifold, quasi-Einstein manifold, special conformally flat, subprojective manifold. 
After long gap the study of a semi-symmetric connection $\bar{\nabla}$ satisfying

$$
\bar{\nabla} g \neq 0
$$

was initiated by Prvanović [9] with the name pseudo-metric semi-symmetric connection and was just followed by Andonie [14].

In 1992, Agashe and Chafle [13] introduced and studied a semi-symmetric nonmetric connection. The semi-symmetric non-metric connections was further developed by several authors such as De and Biswas [18], Biswas, De and Barua [16], De and Kamilya ( [20], [21]) and many others.

In 1967, R.N.Sen and M.C.Chaki [15] studied certain curvature restrictions on a certain kind of conformally flat Riemannian space of class one and they obtained the following expression of the covariant derivative of the curvature tensor :

$$
K_{i j k, l}^{h}=2 \lambda_{l} K_{i j k}^{h}+\lambda_{i} K_{l j k}^{h}+\lambda_{j} K_{i l k}^{h}+\lambda_{k} K_{i j l}^{h}+\lambda^{h} K_{l i j k},
$$

where $K_{i j k}^{h}$ are the components of the curvature tensor K with respect to the LeviCivita connection,

$$
K_{i j k l}=g_{h l} K_{i j k}^{h},
$$

$\lambda_{i}$ is a non-zero covarient vector and "," denotes covarient differentiation with respect to the metric tensor $g_{i j}$.

Later in 1987, M.C.Chaki [10] called a manifold a pseudo symmetric manifold whose curvature tensor satisfies (1.2). In index free notation this can be stated as follows: A non- flat Riemannian manifold $\left(M^{n}, g\right), n \geq 2$ is said to be a pseudo symmetric manifold [10] if its curvature tensor $K$ satisfies the condition

$$
\begin{aligned}
\left(D_{X} K\right)(Y, Z) W= & 2 A(X) K(Y, Z) W+A(Y) K(X, Z) W+A(Z) K(Y, X) W \\
& +A(W) K(Y, Z) X+g(K(Y, Z) W, X) U
\end{aligned}
$$

where $D$ denotes the operator of covarient differentiation with respect to the metric tensor $g$. The 1 - form $\mathrm{A}$ is called the associated 1-form of the manifold . If $A=0$, then the manifold reduces to a symmetric manifold in the sense of Cartan [3]. An $n$-dimensional pseudo symmetric manifold is denoted by $(P S)_{n}$. In this connection we can mention the notion of weakly symmetric manifold introduced by Tamássy and Binh [8]. Such a manifold was denoted by $(W S)_{n}$.

In a recent paper De and Gazi [19] introduced a type of non-flat Riemannian manifold $\left(M^{n}, g\right), n \geq 2$ whose curvature tensor $K$ of type (1,3) satisfies the condition 


$$
\begin{gathered}
\left(D_{X} K\right)(Y, Z) W=[A(X)+B(X)] K(Y, Z) W+A(Y) K(X, Z) W+A(Z) K(Y, X) W \\
+A(W) K(Y, Z) X+g(K(Y, Z) W, X) U,
\end{gathered}
$$

where $\mathrm{A}, \mathrm{U}$ and $\mathrm{D}$ have the meaning already mentioned and B is a non-zero 1-form, $\mathrm{V}$ is a vector field defined by $B(X)=g(X, V), \quad \forall X$.

Such a manifold was called an almost pseudo symmetric manifold and was denoted by $(A P S)_{n}$.

If $B=A$, then from the definitions it follows that $(A P S)_{n}$ deduces to a $(P S)_{n}$. In the same paper the authors constructed two non-trivial examples of $(A P S)_{n}$. It may be mentioned that almost pseudo symmetric manifolds is not a particular case of weakly symmetric manifolds.

Let $\left(M^{n}, g\right),(n>3)$ be a Riemannian manifold admitting a semi-symmetric non-metric connection whose torsion tensor is almost pseudo symmetric, that is, (1.4)

$\left(\nabla_{X} T\right)(Y, Z)=[A(X)+B(X)] T(Y, Z)+A(Y) T(X, Z)+A(Z) T(Y, X)+g(T(Y, Z), X) U$,

where $\mathrm{A}$ and $\mathrm{B}$ are defined earlier.

A non- flat Riemannian manifold $\left(M^{n}, g\right), n \geq 3$ is said to be a quasi-Einstein manifold [11] if its Ricci tensor $\tilde{S}$ of the Levi-Civita connection is of the form

$$
\tilde{S}(X, Y)=a g(X, Y)+b A(X) A(Y),
$$

where $a$ and $b$ are smooth functions of the manifold.

In the present paper we consider a Riemannian manifold admitting a semi- symmetric non-metric connection whose torsion tensor is almost pseudo symmetric.

The paper is organized as follows:

After preliminaries in section 3, we first obtain the expressions of the curvature tensor and the Ricci tensor of the semi-symmetric non-metric connection. In this section we prove that if a Riemannian Manifold admits a semi-symmetric nonmetric connection whose curvature tensor vanishes and the torsion tensor is almost pseudo symmetric with respect to the semi-symmetric non-metric connection, then the manifold becomes a quasi-Einstein manifold. Finally, we deal with a simply connected $(A P S)_{n},(n>3)$ addmitting such a semi-symmetric non-metric connection. 


\section{Preliminaries}

Let $\tilde{r}$ denotes the scalar curvature of the manifold with respect to the LeviCivita connection and $L$ denote the symmetric endomorphism of the tangent space at each point corresponding to the Ricci tensor, that is,

$$
g(L X, Y)=\tilde{S}(X, Y)
$$

for any vector field $X, Y$.

Contracting $Y$ in (1.3), it follows that

$\left(D_{X} \tilde{S}\right)(Z, W)=[A(X)+B(X)] \tilde{S}(Z, W)+A(K(X, Z) W)+A(Z) \tilde{S}(X, W)+A(W) \tilde{S}(X, Z)+g(K(U, Z) W, X)$.

Putting $Z=W=e_{i}$ in $(2.2)$, where $\left\{e_{i}\right\}, \mathrm{i}=1,2,3 \ldots, \mathrm{n}$; is an orthonormal basis of the tangent space at each point of the manifold and taking summation over $i, 1 \leq i \leq n$, we get

$$
d \tilde{r}(X)=[A(X)+B(X)] \tilde{r}+4 A(L X) .
$$

\section{Riemannian Manifolds Admitting a Special Type of the Semi- Symmetric Non-Metric Connection}

Theorem 3.1. If a Riemannian manifold admits a semi-symmetric non-metric connection whose curvature tensor $R$ vanishes and torsion tensor $T$ satisfies (1.4), then the manifold is a quasi-Einstein manifold .

Proof. Let $M$ be an $n$-dimensional Riemannian manifold with Riemannian metric $g$. If $\nabla$ is the semi-symmetric non-metric connection of a Riemannian manifold $M$, then $\nabla$ is given by [13]

$$
\nabla_{X} Y=D_{X} Y+A(Y) X
$$

Let $R$ be the curvature tensor with respect to semi-symmetric non-metric connection. Then $R$ and $K$ are related by [13]

$$
R(X, Y) Z=K(X, Y) Z+\alpha(X, Z) Y-\alpha(Y, Z) X
$$

for all vector fields $X, Y, Z$ on $M$, where $\alpha$ is a $(0,2)$ tensor given by

$$
\alpha(X, Z)=\left(D_{X} A\right)(Z)-A(X) A(Z) .
$$

In this section we consider a Riemannian manifold admitting a semi-symmetric nonmetric connection whose torsion tensor $T$ satisfies (1.4). From (1.1), contracting over $X$, we get

$$
\left(C_{1}^{1} T\right)(Y)=(n-1) A(Y) .
$$


From (3.4), it follows that

$$
\left(\nabla_{X} C_{1}^{1} T\right)(Y)=(n-1)\left(\nabla_{X} A\right)(Y) .
$$

Contracting over $Z$ in (1.4) and using (3.4), we obtain

(3.6) $\left(\nabla_{X} C_{1}^{1} T\right)(Y)=(2 n-3) A(X) A(Y)+(n-1) B(X) A(Y)+A(U) g(X, Y)$.

From (3.5) and (3.6) yields

(3.7) $(n-1)\left(\nabla_{X} A\right)(Y)=(2 n-3) A(X) A(Y)+(n-1) B(X) A(Y)+A(U) g(X, Y)$.

Using (3.1) and (3.3), it follows that

$$
\left(\nabla_{X} A\right)(Y)=\left(D_{X} A\right)(Y)-A(X) A(Y)=\alpha(X, Y) .
$$

Therefore, from (3.7) and (3.8), we have

$$
\alpha(X, Y)=\frac{2 n-3}{n-1} A(X) A(Y)+B(X) A(Y)+\frac{1}{n-1} A(U) g(X, Y) .
$$

Using (3.9) in (3.2) yields

$$
\begin{aligned}
R(X, Y) Z= & K(X, Y) Z+\frac{2 n-3}{n-1} A(X) A(Z) Y+B(X) A(Z) Y+\frac{1}{n-1} A(U) g(X, Z) Y \\
& -\frac{2 n-3}{n-1} A(Y) A(Z) X-B(Y) A(Z) X-\frac{1}{n-1} A(U) g(Y, Z) X .
\end{aligned}
$$

From (3.10), we get

$$
\begin{aligned}
\tilde{R}(X, Y, Z, W)= & \tilde{K}(X, Y, Z, W)+\frac{2 n-3}{n-1} A(X) A(Z) g(Y, W)+B(X) A(Z) g(Y, W) \\
+ & \frac{1}{n-1} A(U) g(X, Z) g(Y, W)-\frac{2 n-3}{n-1} A(Y) A(Z) g(X, W) \\
& -\frac{1}{n-1} A(U) g(Y, Z) g(X, W)-B(Y) A(Z) g(X, W),
\end{aligned}
$$

where $\tilde{R}(X, Y, Z, W)=g(R(X, Y) Z, W)$ and $\tilde{K}(X, Y, Z, W)=g(K(X, Y) Z, W)$.

Putting $X=W=e_{i}$ in (3.11) where $\left\{e_{i}\right\}, 1 \leq i \leq n$ is an orthonormal basis of the tangent space at any point of the manifold $M^{n}$ and then summing over $i$, we 
obtain

$(3.12)$

$$
S(Y, Z)=\tilde{S}(Y, Z)-(2 n-3) A(Y) A(Z)-(n-1) B(Y) A(Z)-A(U) g(Y, Z),
$$

where $S$ be the Ricci tensor with respect to the semi-symmetric non-metric connection.

Proposition 3.1. If a Riemannian manifold admits a semi-symmetric non-metric connection whose torsion tensor is almost pseudo symmetric, then

(i) the curvature tensor of the semi-symmetric non-metric connection is given by (3.10),

(ii) the Ricci tensor of the semi-symmetric non-metric connection is given by (3.12),

(iii) the Ricci tensor $S$ is symmetric if and only if $B(Y) A(Z)=B(Z) A(Y)$.

Suppose

$$
R(X, Y) Z=0 .
$$

Then from the above equation, we have

$$
S(Y, Z)=0
$$

Hence the equation (3.12) reduces to

$$
\tilde{S}(Y, Z)=(2 n-3) A(Y) A(Z)+(n-1) B(Y) A(Z)+A(U) g(Y, Z) .
$$

Since $\tilde{S}$ is symmetric, therefore

Therefore,

$$
B(Y) A(Z)=B(Z) A(Y) .
$$

Putting $Z=U$ in (3.14), it follows that

$$
B(Y)=f A(Y)
$$

where $f=\frac{B(U)}{A(U)}$

Now using (3.17) in (3.15), we obtain

$$
\tilde{S}(Y, Z)=A(U) g(Y, Z)+[(2 n-3)+(n-1) f] A(Y) A(Z) .
$$

Therefore, $\tilde{S}(X, Y)=a g(X, Y)+b A(X) A(Y)$, where $a=A(U)$ and $b=[(2 n-3)+(n-1) f]$.

Hence the proof is completed.

4. Special Conformally Flat $(A P S)_{n}$ Admitting a Special Type of the Semi-Symmetric Non-Metric Connection 
Theorem 4.1. If a $(A P S)_{n}(n>3)$ admits a semi-symmetric non-metric connection whose torsion tensor is almost pseudo symmetric and the curvature tensor of the semi-symmetric non-metric connection vanishes, then the manifold is a particular kind of a special conformally flat manifold, namely a subprojective manifold.

Proof. Chen and Yano [2] introduced the notion of a special conformally flat manifold which generalizes the notion of a subprojective manifold. A conformally flat manifold is called a special conformally flat manifold if the tensor $H$ of type $(0,2)$ defined by

$$
H(X, Y)=-\frac{1}{n-2} \tilde{S}(X, Y)+\frac{\tilde{r}}{2(n-1)(n-2)} g(X, Y),
$$

is expressible in the form

$$
H(X, Y)=-\frac{\alpha^{2}}{2} g(X, Y)+\beta\left(D_{X} \alpha\right)\left(D_{Y} \alpha\right),
$$

where $\alpha$ and $\beta$ are two scalars such that $\alpha$ is positive. In particular, if $\beta$ is a function of $\alpha$ then the special conformally flat manifold is called a subprojective manifold [5].

Let us consider $(A P S)_{n}$ admitting a semi-symmetric non-metric connection whose torsion tensor is almost pseudo symmetric and the curvature tensor of the semisymmetric non-metric connection vanishes.

Using (3.16) in (4.1), we get

$$
H(X, Y)=\frac{\tilde{r}-2(n-1) A(U)}{2(n-1)(n-2)} g(X, Y)-\frac{2 n-3+f(n-1)}{n-2} A(X) A(Y) .
$$

Now, put

$$
\alpha^{2}=-\frac{\tilde{r}-2(n-1) A(U)}{(n-1)(n-2)} .
$$

From (3.16), we get

$$
\tilde{r}=(n-1)(3+f) A(U), n \geq 3 .
$$

Since $\tilde{r} \neq 0$, it follows that $\alpha^{2}$ will be positive provided that $\tilde{r}<0$.

From (3.16) and (2.1), it follows that

$$
L Y=[2 n-3+(n-1) f] A(Y) U+A(U) Y .
$$

From (4.5), we obtain

$$
A(L Y)=(n-1)(2+f) A(U) A(Y) .
$$

Using (4.6) and (3.15) in (2.3), we have

$$
d \tilde{r}(X)=[(1+f) \tilde{r}+4(n-1)(2+f) A(U)] A(X) .
$$


Let us take the covariant derivative of both side of (4.3) with respect to $X$ and using (4.7), we obtain

$$
D_{X} \alpha=-\frac{(1+f) \tilde{r}+4(n-1)(2+f) A(U)}{2(n-1)(n-2) \alpha} A(X) .
$$

From (4.8), we have

$$
A(X)=-\frac{2(n-1)(n-2) \alpha}{(1+f) \tilde{r}+4(n-1)(2+f) A(U)} D_{X} \alpha .
$$

Thus, due to (4.3), (4.9) and (4.2) can be expressed in the form

$$
H(X, Y)=-\frac{\alpha^{2}}{2} g(X, Y)+\beta\left(D_{X} \alpha\right)\left(D_{Y} \alpha\right),
$$

where

$$
\beta=-\frac{4(2 n-3)+(n-1) f)(n-1)^{2}(n-2)}{[(1+f) \tilde{r}+4(n-1)(2+f) A(U)]^{2}} \alpha^{2} .
$$

In virtue of (4.10), we deduce that $\beta$ is a function of $\alpha$. Thus the theorem is proved.

Corollary 4.1.([2]) Every simply connected subprojective space can be isometrically immersed in a Euclidean space as a hypersurface.

Moreover, using this Corollary, we can also state the following theorem:

Theorem 4.2. If a simply connected $(A P S)_{n}(n>3)$ admits a semi-symmetric non-metric connection whose torsion tensor is almost pseudo symmetric and the curvature tensor of the semi-symmetric non-metric connection vanishes, then the manifold can be isometrically immersed in a Euclidean space $E^{n+1}$ as a hypersurface.

\section{References}

[1] A. Friedmann and J. A. Schouten, Über die Geometric der halbsymmetrischen Übertragung, Math., Zeitschr., 21(1924), 211-223.

[2] B. Y. Chen and K. Yano, Special conformally flat spaces and canal hypersurfaces, Tohoku Math. J., 25(1973), 177-184.

[3] E. Cartan, Sur une classe remarquable d'espaces de Riemannian, Bull. Soc. Math. France, 54(1926), 214-264.

[4] H. A. Hayden, Subspaces of space with torsion, Proc. London Math. Soc., 34(1932), $27-50$. 
[5] J. A. Schouten, Ricci-Calculus, $2^{\text {nd }}$ Edition, Springer-Verlag, Berlin-GöttingenHeidelberg, 1954.

[6] K. Yano, On semi-symmetric connection, Revue Roumaine de Math. Pure et Appliques, 15(1970), 1570-1586.

[7] K. Amur and S. S. Pujar, On submanifolds of a Riemannian manifold admitting a metric semi-symmetric connection, Tensor, N. S., 32(1978), 35-38.

[8] L. Tamássy and T. Q. Binh, On weakly symmetric and weakly projective symmetric Riemannian manifolds, Colloq. Math. Soc. J. Bolyai, 50(1989), 663-670.

[9] M. Prvanović, On pseudo metric semi-symmetric connections, Pub. De L Institut Math., Nouvelle serie, 18(32)(1975), 157-164.

[10] M. C. Chaki, On pseudo symmetric manifolds, Analele stiintifice Ale Universitatii, "AL. I. CUSA" DIN IASI, 33(1987), 53-58.

[11] M. C. Chaki and R. K. Maity, On quasi-Einstein manifolds, Publ. Math. Debrecen, 57(2000), 297-306.

[12] M. C. Chaki and A. Konar, On a type of semi-symmetric connection on a Riemannian manifold, J. Pure Math., Calcutta University, (1981), 77-80.

[13] N. S. Agashe and M. R. Chafle, A semi-symmetric non-metric connection on a Riemannian Manifold, Indian J. Pure Appl. Math., 23(6)(1992), 399-409.

[14] O. C. Andonie, On semi-symmetric non-metric connection on a Riemannian manifold, Ann. Fac. Sci. De Kinshasa, Zaire Sect. Math. Phys., 2(1976).

[15] R. N. Sen and M. C. Chaki, On curvature restrictions of a certain kind of conformally flat Riemannian space of class one, Proc. Nat. Inst. Sci. India, 33(1967), 100-102.

[16] S. C. Biswas, U. C. De and B. Barua, Semi-symmetric non-metric connection in an SP-Sasakian manifold, J. Pure Math. Calcutta University, 13(1996), 13-18.

[17] U. C. De, On a type of semi-symmetric connection on a Riemannian manifold, Indian J. Pure Appl. Math., 21(4), April 1990, 334-338.

[18] U. C. De and S. C. Biswas, On a type of semi-symmetric non-metric connection on a Riemannian manifold, Ganita, 48(1997), 91-94.

[19] U. C. De and A. K. Gazi, On almost pseudo symmetric manifolds, Ann. Univ. Sci. Budapest, Sec.Math., 51(2008), 53-68.

[20] U. C. De and D. Kamilya, On a type of semi-symmetric non-metric connection on a Riemannian manifold, Istanbul Üniv. Fen. Fak. Mat. Der., 53(1994), 37-41.

[21] U. C. De and D. Kamilya, Hypersurfaces of a Riemannian manifold with semisymmetric non-metric connection, J. Indian Inst. Sci., 75(1995), 707-710. 